\title{
Computational Study of Actinyl Ions Complexation with Dipyriamethyrin Macrocyclic Ligands
}

Elumalai Varathan, Yang Gao and Georg Schreckenbach*

Department of Chemistry, University of Manitoba, Winnipeg, Manitoba, R3T 2N2, Canada.

\section{CONTENT CAPTIONS}

Table S1 Calculated bond lengths $(\AA)$, bond angles $\left(^{\circ}\right)$, bond orders (B.O), NPA charges and spin density on the actinide atom (atomic units), and actinyl stretching vibrational frequencies $\left(\mathrm{cm}^{-1}\right)$ of the bare actinyl (VI, V) ions at the PBE/AE-TZP level of theory in gas phase.

Table S2 Calculated bond lengths $(\AA)$ and bond angles (deg) of the actinyl (VI and V) complexes of macrocycle with $\mathrm{An}=\mathrm{U}, \mathrm{Np}, \mathrm{Pu}$ and $\mathrm{Am}$ at the PBE/TZP level of theory using Priroda in gas phase.

Table S3 Calculated bond lengths $(\AA)$ and bond angles (deg) of the actinyl (VI and V) complexes of macrocycle with $\mathrm{An}=\mathrm{U}, \mathrm{Np}, \mathrm{Pu}$ and $\mathrm{Am}$ at the PBE/TZP level of theory using ADF and ZORA in $\mathrm{CH}_{2} \mathrm{Cl}_{2}$ solvent.

Table S4 Calculated nitrogen NPA charges for the free ligand anion and upon actinyl (VI and V) complexation at the PBE/TZP level of theory using ADF.

Table S5 Calculated Mayer bond orders of the Actinyl (VI and V) Complexes (An=U, Np, Pu and Am).

Table S6 Calculated NPA valence populations for An (VI and V) ions complexed with the dipyriamethyrin ligand.

Table S7 Natural Orbital Population of An (An =U, Np, Pu and Am) centers in the bare Actinyl (VI and V) cations.

Table S8 Natural electron configurations of the actinide in the actinyl (VI and V) complexes.

Table S9 Natural electron configurations of the actinide in the free actinyl (VI and V) cations.

Table S10 Calculated bond lengths $(\AA)$ and bond angles $\left(^{\circ}\right)$ of the actinyl (VI, V) dichlorides in $\mathrm{CH}_{2} \mathrm{Cl}_{2}$

Table S11 Atomic orbital contribution (\%) to specific molecular orbitals (MOs) in the $\left[\mathrm{UO}_{2} \mathrm{~L}\right]$ complex.

Figure S1 Optimized geometries of $\left[\mathrm{AnO}_{2}(\mathrm{~L})\right]^{0 / 1-}(\mathrm{An}=\mathrm{U}, \mathrm{Np}, \mathrm{Pu}, \mathrm{Am})$ complexes.

Figure S2 Electron donation into the valence d ( $\square)$ and f ( $\square$ ) orbitals in An (VI) (left) and An (V) (right) complexes. 
Figure S3 Formal and actual spin densities on the An (U, Np, Pu and Am) metal in the actinyl(VI) (left) and actinyl (V) (right) complexes.

Figure S4 Optimized geometries of actinyl dichlorides at PBE/TZP level of theory in $\mathrm{CH}_{2} \mathrm{Cl}_{2}$.

Figure S5 Molecular orbital energy $(\mathrm{eV})$ diagram of $\mathrm{UO}_{2} \mathrm{~L}$ and its two fragments. 
Table S1: Calculated bond lengths $(\AA)$, bond angles $\left({ }^{\circ}\right)$, bond orders (B.0), NPA charges, spin density on the actinide atom (atomic units), and actinyl stretching vibrational frequencies $\left(\mathrm{cm}^{-1}\right)$ of the bare actinyl (VI, V) ions at the PBE/ZORA-TZP level of theory in gas phase.

\begin{tabular}{lcccccccc}
\hline & $\mathrm{An}^{\mathrm{a}}=0$ & $0=\mathrm{An}=0$ & $\mathrm{~B} .0$ & $\mathrm{q}(\mathrm{An})$ & $\mathrm{q}(\mathrm{O})$ & Spin on $\mathrm{An}$ & $\boldsymbol{v}_{\text {symm }}\left(\mathrm{AnO}_{2}\right)$ & $\boldsymbol{v}_{\text {asymm }}\left(\mathrm{AnO}_{2}\right)$ \\
\hline $\mathrm{UO}_{2}{ }^{2+}$ & 1.720 & 180.0 & 2.272 & 2.686 & -0.343 & 0.0 & 994 & 1096 \\
$\mathrm{UO}_{2}{ }^{+}$ & 1.768 & 180.0 & 2.062 & 2.202 & -0.601 & 1.085 & 926 & 1005 \\
$\mathrm{NpO}_{2}{ }^{2+}$ & 1.719 & 180.0 & 2.244 & 2.546 & -0.273 & 1.151 & 968 & 1085 \\
$\mathrm{NpO}_{2}{ }^{+}$ & 1.741 & 180.0 & 2.085 & 2.086 & -0.543 & 2.198 & 919 & 1001 \\
$\mathrm{PuO}_{2}{ }^{2+}$ & 1.690 & 180.0 & 2.264 & 2.341 & -0.171 & 2.254 & 978 & 1097 \\
$\mathrm{PuO}_{2}{ }^{+}$ & 1.740 & 180.0 & 2.104 & 1.921 & -0.461 & 3.315 & 894 & 990 \\
$\mathrm{AmO}_{2}{ }^{2+}$ & 1.698 & 180.0 & 2.210 & 2.242 & -0.121 & 3.452 & 915 & 1053 \\
$\mathrm{AmO}_{2}{ }^{+}$ & 1.745 & 180.0 & 2.070 & 1.821 & -0.410 & 4.500 & 845 & 963 \\
\hline
\end{tabular}

${ }^{\mathrm{a} A n}=\mathrm{U}, \mathrm{Np}, \mathrm{Pu}$ and Am. 
Table S2: Calculated bond lengths (Å) and bond angles (deg) of the actinyl (VI and V) complexes at the PBE/TZP level of theory using Priroda in gas phase.

\begin{tabular}{|c|c|c|c|c|c|c|c|c|c|}
\hline & $\mathrm{UO}_{2} \mathrm{~L}$ & $\mathrm{NpO}_{2} \mathrm{~L}$ & $\mathrm{PuO}_{2} \mathrm{~L}$ & $\mathrm{AmO}_{2} \mathrm{~L}$ & $\mathrm{UO}_{2} \mathrm{~L}^{-}$ & $\mathrm{NpO}_{2} \mathrm{~L}^{-}$ & $\mathrm{PuO}_{2} \mathrm{~L}^{-}$ & $\mathrm{AmO}_{2} \mathrm{~L}^{-}$ & $\mathrm{UO}_{2} \mathrm{~L}(\mathrm{Exp})^{\mathrm{a}}$ \\
\hline \multicolumn{10}{|c|}{ Bond Lengths } \\
\hline An-N1 & 2.756 & 2.749 & 2.755 & 2.746 & 2.789 & 2.794 & 2.825 & 2.883 & 2.712 \\
\hline An-N2 & 2.651 & 2.639 & 2.674 & 2.647 & 2.681 & 2.717 & 2.753 & 2.791 & 2.600 \\
\hline An-N3 & 2.651 & 2.637 & 2.665 & 2.646 & 2.681 & 2.720 & 2.756 & 2.797 & 2.615 \\
\hline An-N4 & 2.756 & 2.749 & 2.758 & 2.746 & 2.789 & 2.794 & 2.824 & 2.883 & 2.701 \\
\hline An-N5 & 2.651 & 2.639 & 2.668 & 2.647 & 2.681 & 2.720 & 2.754 & 2.793 & 2.635 \\
\hline An-N6 & 2.651 & 2.637 & 2.671 & 2.646 & 2.682 & 2.722 & 2.756 & 2.797 & 2.622 \\
\hline$A n=0$ & 1.789 & 1.772 & 1.769 & 1.758 & 1.807 & 1.801 & 1.794 & 1.795 & 1.772 \\
\hline \multicolumn{10}{|l|}{ Bond Angles } \\
\hline $0=A n=0$ & 180.0 & 180.0 & 179.5 & 180.0 & 179.7 & 180.0 & 180.0 & 180.0 & 179.4 \\
\hline $\mathrm{N} 1-\mathrm{An}-\mathrm{N} 2$ & 58.9 & 59.0 & 59.0 & 59.1 & 58.6 & 58.6 & 58.3 & 57.7 & 59.7 \\
\hline $\mathrm{N} 2-\mathrm{An}-\mathrm{N} 3$ & 65.7 & 65.8 & 65.4 & 65.6 & 65.8 & 65.6 & 65.4 & 65.8 & 66.3 \\
\hline N3-An-N4 & 59.1 & 59.1 & 59.1 & 59.3 & 58.8 & 58.8 & 58.5 & 58.0 & 59.0 \\
\hline $\mathrm{N} 4-\mathrm{An}-\mathrm{N} 5$ & 58.9 & 59.0 & 58.9 & 59.1 & 58.7 & 58.6 & 58.3 & 57.7 & 59.6 \\
\hline N5-An-N6 & 65.7 & 65.8 & 65.5 & 65.6 & 65.8 & 65.5 & 65.4 & 65.8 & 65.4 \\
\hline N6-An-N1 & 59.1 & 59.1 & 59.1 & 59.3 & 58.9 & 58.7 & 58.5 & 57.9 & 58.8 \\
\hline
\end{tabular}

a Chem. Commun., 2017, 53, 4981-4984. 
Table S3: Calculated bond lengths $(\AA)$ and bond angles (deg) of the actinyl (VI and V) complexes at the PBE/TZP level of theory using ADF and ZORA in $\mathrm{CH}_{2} \mathrm{Cl}_{2}$ solvent.

\begin{tabular}{lcccccccc}
\hline & $\mathrm{UO}_{2} \mathrm{~L}$ & $\mathrm{NpO}_{2} \mathrm{~L}$ & $\mathrm{PuO}_{2} \mathrm{~L}$ & $\mathrm{AmO}_{2} \mathrm{~L}$ & $\mathrm{UO}_{2} \mathrm{~L}^{-}$ & $\mathrm{NpO}_{2} \mathrm{~L}^{-}$ & $\mathrm{PuO}_{2} \mathrm{~L}^{-}$ & $\mathrm{AmO}_{2} \mathrm{~L}^{-}$ \\
\hline Bond Lengths & & & & & & & & \\
\hline An-N1/N4 & 2.750 & 2.753 & 2.769 & 2.758 & 2.794 & 2.822 & 2.831 & 2.884 \\
An-N2/N5 & 2.640 & 2.632 & 2.690 & 2.658 & 2.688 & 2.728 & 2.751 & 2.783 \\
An-N3/N6 & 2.639 & 2.631 & 2.690 & 2.656 & 2.685 & 2.728 & 2.748 & 2.778 \\
An=0 & 1.807 & 1.788 & 1.789 & 1.763 & 1.839 & 1.834 & 1.820 & 1.816 \\
\hline Bond Angles & & & & & & & & \\
\hline O=An=0 & 180.0 & 180.0 & 180.0 & 180.0 & 180.0 & 180.0 & 180.0 & 180.0 \\
N1-An-N2 & 59.0 & 58.9 & 58.8 & 59.0 & 58.6 & 58.4 & 58.2 & 57.7 \\
N2-An-N3 & 65.6 & 65.8 & 65.2 & 65.6 & 65.7 & 65.6 & 65.3 & 65.7 \\
N3-An-N4 & 59.2 & 59.1 & 59.0 & 59.2 & 58.8 & 58.6 & 58.5 & 58.0 \\
N4-An-N5 & 59.0 & 58.9 & 58.8 & 59.0 & 58.6 & 58.2 & 58.2 & 57.7 \\
N5-An-N6 & 65.6 & 65.8 & 65.2 & 65.6 & 65.7 & 65.4 & 65.3 & 65.7 \\
N6-An-N1 & 59.2 & 59.1 & 59.0 & 59.2 & 58.8 & 58.5 & 58.5 & 58.0 \\
\hline
\end{tabular}


Table S4: Calculated nitrogen NPA charges for the free ligand anion and upon actinyl (VI and V) complexation at the PBE/TZP level of theory using ADF.

\begin{tabular}{|c|c|c|c|c|c|c|c|c|}
\hline & $\mathrm{UO}_{2} \mathrm{~L}$ & $\mathrm{NpO}_{2} \mathrm{~L}$ & $\mathrm{PuO}_{2} \mathrm{~L}$ & $\mathrm{AmO}_{2} \mathrm{~L}$ & $\mathrm{UO}_{2} \mathrm{~L}^{-}$ & $\mathrm{NpO}_{2} \mathrm{~L}^{-}$ & $\mathrm{PuO}_{2} \mathrm{~L}^{-}$ & $\mathrm{AmO}_{2} \mathrm{~L}^{-}$ \\
\hline \multicolumn{9}{|c|}{ Within the actinyl complexes } \\
\hline $\mathrm{N} 1 / \mathrm{N} 4$ & -0.358 & -0.358 & -0.369 & -0.363 & -0.362 & -0.369 & -0.370 & -0.386 \\
\hline N2/N5 & -0.397 & -0.384 & -0.402 & -0.400 & -0.405 & -0.403 & -0.402 & -0.418 \\
\hline N3/N6 & -0.398 & -0.385 & -0.403 & -0.401 & -0.406 & -0.403 & -0.403 & -0.421 \\
\hline \multicolumn{9}{|c|}{ Free ligand anion } \\
\hline $\mathrm{N} 1 / \mathrm{N} 4$ & -0.342 & & & & & & & \\
\hline N2/N5 & -0.352 & & & & & & & \\
\hline N3/N6 & -0.356 & & & & & & & \\
\hline
\end{tabular}

Table S5: Calculated Mayer bond orders of the Actinyl (VI and V) Complexes (An=U, Np, $\mathrm{Pu}$ and $\mathrm{Am}$ ).

\begin{tabular}{ccccccccc}
\hline & $\mathrm{UO}_{2} \mathrm{~L}$ & $\mathrm{NpO}_{2} \mathrm{~L}$ & $\mathrm{PuO}_{2} \mathrm{~L}$ & $\mathrm{AmO}_{2} \mathrm{~L}$ & $\mathrm{UO}_{2} \mathrm{~L}^{-}$ & $\mathrm{NpO}_{2} \mathrm{~L}^{-}$ & $\mathrm{PuO}_{2} \mathrm{~L}^{-}$ & $\mathrm{AmO}_{2} \mathrm{~L}^{-}$ \\
\hline $\mathrm{An}=\mathrm{O}$ & 1.931 & 1.923 & 1.897 & 1.839 & 1.902 & 1.883 & 1.875 & 1.851 \\
$\mathrm{An}-\mathrm{N} 1 / \mathrm{N}^{\mathrm{a}}$ & 0.257 & 0.252 & 0.250 & 0.218 & 0.226 & 0.203 & 0.163 & 0.104 \\
$\mathrm{An}^{\mathrm{N} 2} / \mathrm{N5}^{\mathrm{a}}$ & 0.342 & 0.342 & 0.274 & 0.279 & 0.321 & 0.257 & 0.203 & 0.167 \\
$\mathrm{An}-\mathrm{N} 3 / \mathrm{N6}^{\mathrm{a}}$ & 0.342 & 0.341 & 0.272 & 0.278 & 0.319 & 0.254 & 0.201 & 0.166 \\
\hline
\end{tabular}


Table S6: Calculated natural orbital populations for An (VI and V) ions complexed with the dipyriamethyrin ligand.

\begin{tabular}{cccccccc} 
& \multicolumn{3}{c}{$\left[\mathrm{AnO}_{2} \mathrm{~L}\right]$} & & \multicolumn{3}{c}{$\left[\mathrm{AnO}_{2} \mathrm{~L}\right]^{-1}$} \\
\hline & $6 \mathrm{~d}^{\mathrm{a}}$ & $5 \mathrm{f}$ & $\Delta 5 \mathrm{f}^{\mathrm{b}}$ & & $6 \mathrm{~d}^{\mathrm{a}}$ & $5 \mathrm{f}$ & $\Delta 5 \mathrm{f}^{\mathrm{b}}$ \\
\hline $\mathrm{U}$ & 1.60 & 2.43 & 2.43 & 1.51 & 2.86 & 1.86 \\
$\mathrm{~Np}$ & 1.53 & 2.99 & 1.99 & 1.36 & 4.19 & 2.19 \\
$\mathrm{Pu}$ & 1.41 & 4.62 & 2.62 & & 1.25 & 5.40 & 2.40 \\
$\mathrm{Am}$ & 1.38 & 6.33 & 3.33 & & 1.14 & 6.39 & 2.39
\end{tabular}

aThe formal An $6 \mathrm{~d}$ population is 0 , hence $6 \mathrm{~d}$ is equivalent to $\Delta 6 \mathrm{~d}$.

${ }^{\mathrm{b}} \Delta 5 \mathrm{f}$ indicates the change in $5 \mathrm{f}$ population resulting from complexation, i.e. $\Delta 5 \mathrm{f}$ is the $5 \mathrm{f}$ population minus the formal $5 \mathrm{f}$ population.

Table S7: Natural Orbital Population of $\mathrm{An}(\mathrm{An}=\mathrm{U}, \mathrm{Np}, \mathrm{Pu}$ and $\mathrm{Am})$ centers in the bare Actinyl (VI and V) cations.

\begin{tabular}{|c|c|c|c|c|c|c|}
\hline & \multirow{2}{*}{$\begin{array}{c}\text { NPA charge } \\
\mathrm{An}\end{array}$} & \multicolumn{4}{|c|}{ Natural orbital population } & \multirow{2}{*}{$\frac{\text { Spin density }}{\mathrm{An}}$} \\
\hline & & $5 f$ & $6 d$ & $7 \mathrm{~s}$ & $7 p$ & \\
\hline$\left[\mathrm{UO}_{2}\right]^{2+}$ & 2.69 & 2.55 & 1.03 & 0.05 & 0.00 & 0.00 \\
\hline$\left[\mathrm{NpO}_{2}\right]^{2+}$ & 2.55 & 2.52 & 0.93 & 0.03 & 0.00 & 1.15 \\
\hline$\left[\mathrm{PuO}_{2}\right]^{2+}$ & 2.34 & 4.97 & 0.93 & 0.04 & 0.00 & 2.25 \\
\hline$\left[\mathrm{AmO}_{2}\right]^{2+}$ & 2.24 & 5.25 & 0.87 & 0.03 & 0.00 & 3.45 \\
\hline$\left[\mathrm{UO}_{2}\right]^{+}$ & 2.20 & 3.04 & 0.94 & 0.05 & 0.00 & 1.09 \\
\hline$\left[\mathrm{NpO}_{2}\right]^{+}$ & 2.09 & 4.21 & 0.91 & 0.05 & 0.00 & 2.20 \\
\hline$\left[\mathrm{PuO}_{2}\right]^{+}$ & 1.92 & 5.42 & 0.87 & 0.04 & 0.00 & 3.31 \\
\hline$\left[\mathrm{AmO}_{2}\right]^{+}$ & 1.82 & 6.55 & 0.81 & 0.03 & 0.00 & 4.50 \\
\hline
\end{tabular}


Table S8: Natural electron configurations of the actinide in the actinyl (VI and V) complexes

\begin{tabular}{|c|c|c|c|c|c|c|c|c|}
\hline \multirow[t]{3}{*}{ Complex } & \multicolumn{8}{|c|}{ Natural electron configuration } \\
\hline & \multicolumn{4}{|c|}{ alpha } & \multicolumn{4}{|c|}{ beta } \\
\hline & $5 f$ & $6 \mathrm{~d}$ & $7 \mathrm{~s}$ & $7 p$ & $5 f$ & $6 \mathrm{~d}$ & $7 \mathrm{~s}$ & $7 p$ \\
\hline $\mathrm{UO}_{2} \mathrm{~L}$ & - & - & - & - & - & - & - & - \\
\hline $\mathrm{NpO}_{2} \mathrm{~L}$ & 2.61 & 0.78 & 0.10 & 0.01 & 1.48 & 0.75 & 0.09 & 0.01 \\
\hline $\mathrm{PuO}_{2} \mathrm{~L}$ & 3.91 & 0.73 & 0.10 & 0.01 & 1.36 & 0.67 & 0.09 & 0.01 \\
\hline $\mathrm{AmO}_{2} \mathrm{~L}$ & 4.96 & 0.73 & 0.10 & 0.01 & 1.37 & 0.65 & 0.09 & 0.01 \\
\hline $\mathrm{UO}_{2} \mathrm{~L}^{-}$ & 1.64 & 0.76 & 0.09 & 0.01 & 1.23 & 0.75 & 0.09 & 0.01 \\
\hline $\mathrm{NpO}_{2} \mathrm{~L}^{-}$ & 3.01 & 0.70 & 0.09 & 0.01 & 1.18 & 0.66 & 0.09 & 0.01 \\
\hline $\mathrm{PuO}_{2} \mathrm{~L}^{-}$ & 4.28 & 0.66 & 0.09 & 0.00 & 1.11 & 0.59 & 0.08 & 0.01 \\
\hline $\mathrm{AmO}_{2} \mathrm{~L}^{-}$ & 5.38 & 0.61 & 0.09 & 0.00 & 1.01 & 0.53 & 0.08 & 0.01 \\
\hline
\end{tabular}

Table S9: Natural electron configurations of the actinide in the free actinyl (VI and V) cations.

\begin{tabular}{lccccccccc}
\hline Complex & \multicolumn{8}{c}{ Natural electron configuration } \\
\hline & \multicolumn{9}{c}{ alpha } \\
\hline & $5 \mathrm{f}$ & $6 \mathrm{~d}$ & $7 \mathrm{~s}$ & $7 \mathrm{p}$ & $5 \mathrm{c}$ & $6 \mathrm{~d}$ & $7 \mathrm{~s}$ & $7 \mathrm{p}$ \\
\hline$\left[\mathrm{UO}_{2}\right]^{2+}$ & - & - & - & - & - & - & - & - \\
{$\left[\mathrm{NpO}_{2}\right]^{2+}$} & 2.43 & 0.47 & 0.02 & 0.00 & 1.30 & 0.45 & 0.01 & 0.00 \\
{$\left[\mathrm{PuO}_{2}\right]^{2+}$} & 3.60 & 0.48 & 0.02 & 0.00 & 1.37 & 0.45 & 0.02 & 0.00 \\
{$\left[\mathrm{AmO}_{2}\right]^{2+}$} & 4.76 & 0.46 & 0.02 & 0.00 & 1.36 & 0.41 & 0.01 & 0.00 \\
{$\left[\mathrm{UO}_{2}\right]^{+}$} & 2.06 & 0.48 & 0.03 & 0.00 & 0.99 & 0.46 & 0.02 & 0.00 \\
{$\left[\mathrm{NpO}_{2}\right]^{+}$} & 3.18 & 0.48 & 0.02 & 0.00 & 1.03 & 0.43 & 0.02 & 0.00 \\
{$\left[\mathrm{PuO}_{2}\right]^{+}$} & 4.34 & 0.46 & 0.02 & 0.00 & 1.08 & 0.40 & 0.02 & 0.00 \\
{$\left[\mathrm{AmO}_{2}\right]^{+}$} & 5.50 & 0.44 & 0.02 & 0.00 & 1.05 & 0.37 & 0.02 & 0.00 \\
\hline
\end{tabular}


Table S10: Calculated bond lengths $(\AA)$ and bond angles $\left(^{\circ}\right)$ of the actinyl (VI, V) dichlorides in $\mathrm{CH}_{2} \mathrm{Cl}_{2}{ }^{\text {a }}$

\begin{tabular}{lcccccccc} 
& $\mathrm{UO}_{2} \mathrm{Cl}_{2}$ & $\mathrm{NpO}_{2} \mathrm{Cl}_{2}$ & $\mathrm{PuO}_{2} \mathrm{Cl}_{2}$ & $\mathrm{AmO}_{2} \mathrm{Cl}_{2}$ & $\mathrm{UO}_{2} \mathrm{Cl}_{2}^{-}$ & $\mathrm{NpO}_{2} \mathrm{Cl}_{2}^{-}$ & $\mathrm{PuO}_{2} \mathrm{Cl}_{2}^{-}$ & $\mathrm{AmO}_{2} \mathrm{Cl}_{2}^{-}$ \\
\hline $\mathrm{An}=\mathrm{O}$ & 1.790 & 1.770 & 1.764 & 1.751 & 1.849 & 1.857 & 1.803 & 1.803 \\
$\mathrm{An}-\mathrm{Cl}$ & 2.570 & 2.581 & 2.557 & 2.591 & 2.712 & 2.708 & 2.714 & 2.720 \\
$\mathrm{O}=\mathrm{An}=0$ & 173.2 & 179.8 & 176.0 & 179.9 & 172.4 & 174.9 & 177.8 & 178.8 \\
$\mathrm{Cl}-\mathrm{An}-$ & 92.4 & 179.6 & 91.8 & 177.4 & 102.0 & 158.2 & 105.1 & 107.2 \\
$\mathrm{Cl}$ & & & & & & & &
\end{tabular}

aPBE/ZORA-TZP level of theory.

Table S11: Atomic Orbital Contribution (\%) to Specific Molecular Orbitals (MOs) in the $\left[\mathrm{UO}_{2} \mathrm{~L}\right]$ complex.

\begin{tabular}{|c|c|c|}
\hline MOs & U & $\mathrm{N}$ \\
\hline HOMO-4 & $1.40,5 \mathrm{f}_{\mathrm{z}}{ }^{3}$ & $\begin{array}{l}\mathrm{N} 3,10.46\left(2 \mathrm{P}_{\mathrm{z}}\right), 3.19\left(2 \mathrm{P}_{\mathrm{x}}\right), 2.16\left(2 \mathrm{P}_{\mathrm{y}}\right) ; \\
\mathrm{N} 2,8.77\left(2 \mathrm{p}_{\mathrm{z}}\right), 4.49\left(2 \mathrm{P}_{\mathrm{y}}\right) ; \mathrm{N} 1,1.96\left(2 \mathrm{p}_{\mathrm{z}}\right)\end{array}$ \\
\hline HOMO-8 & $1.30,5 f_{x}$ & $\mathrm{~N} 3,18.22\left(2 \mathrm{P}_{\mathrm{z}}\right) ; \mathrm{N} 1,1.22\left(2 \mathrm{P}_{\mathrm{z}}\right)$ \\
\hline HOMO-10 & $1.09,6 \mathrm{~d}_{\mathrm{xy}}$ & $\mathrm{N} 1,22.60\left(2 \mathrm{p}_{\mathrm{z}}\right), 15.32\left(2 \mathrm{p}_{\mathrm{x}}\right)$ \\
\hline HOMO-11 & $1.45,5 \mathrm{f}_{\mathrm{z}}{ }^{3}$ & $\mathrm{~N} 2,18.80\left(2 \mathrm{p}_{\mathrm{y}}\right) ; \mathrm{N} 3,17.43\left(2 \mathrm{p}_{\mathrm{x}}\right)$ \\
\hline HOMO-12 & $1.49,6 \mathrm{~d}_{\mathrm{xy}} ; 1.16,6 \mathrm{~d}_{\mathrm{x}^{2}-\mathrm{y}^{2}}$ & $\mathrm{~N} 3,6.23\left(2 \mathrm{p}_{\mathrm{x}}\right) ; \mathrm{N} 2,5.51\left(2 \mathrm{p}_{\mathrm{y}}\right), \mathrm{N} 1,1.95\left(2 \mathrm{p}_{\mathrm{z}}\right)$ \\
\hline HOMO-13 & $3.94,6 \mathrm{~d}_{\mathrm{xy}}$ & $\begin{array}{l}\mathrm{N} 3,10.97\left(2 \mathrm{p}_{\mathrm{x}}\right) ; \mathrm{N} 3,3.80\left(2 \mathrm{p}_{\mathrm{y}}\right) ; \mathrm{N} 2,6.90\left(2 \mathrm{p}_{\mathrm{y}}\right) ; \\
\mathrm{N} 1,2.93\left(2 \mathrm{p}_{\mathrm{x}}\right) ; \mathrm{N} 1,2.08\left(2 \mathrm{p}_{\mathrm{z}}\right)\end{array}$ \\
\hline HOMO-14 & $4.23,6 \mathrm{~d}_{\mathrm{x} 2-\mathrm{y} 2} ; 1.54,5 \mathrm{f}_{\mathrm{z}}{ }^{3}$ & $\mathrm{~N} 2,12.97\left(2 \mathrm{P}_{\mathrm{x}}\right) ; \mathrm{N} 2,11.42\left(2 \mathrm{P}_{\mathrm{z}}\right) ; \mathrm{N} 1,6.4\left(2 \mathrm{p}_{\mathrm{y}}\right)$ \\
\hline HOMO-16 & $1.47,6 \mathrm{~d}_{\mathrm{z}}^{2}$ & $\mathrm{~N} 2,11.95\left(2 \mathrm{p}_{\mathrm{y}}\right) ; \mathrm{N} 2,9.82\left(2 \mathrm{P}_{\mathrm{x}}\right) ; \mathrm{N} 1,5.02\left(2 \mathrm{p}_{\mathrm{y}}\right)$ \\
\hline HOMO-18 & $1.34,5 \mathrm{f}_{\mathrm{z}}^{3}$ & $\mathrm{~N} 1,11.07\left(2 \mathrm{p}_{\mathrm{x}}\right) ; \mathrm{N} 1,3.14\left(2 \mathrm{p}_{\mathrm{z}}\right) ; \mathrm{N} 1,1.47\left(2 \mathrm{p}_{\mathrm{y}}\right)$ \\
\hline HOMO-19 & $16.80,5 \mathrm{f}_{\mathrm{z}}^{2} ; 1.07,5 \mathrm{f}_{\mathrm{z}}^{2} \mathrm{x}$ & $\mathrm{N} 3,1.83\left(2 \mathrm{p}_{\mathrm{x}}\right)$ \\
\hline HOMO-21 & $3.81,5 \mathrm{f}_{\mathrm{z}}^{2} \mathrm{y} ; 1.64,5 \mathrm{f}_{\mathrm{z}}^{2} \mathrm{x}$ & $\mathrm{N} 3,3.18\left(2 \mathrm{p}_{\mathrm{y}}\right) ; \mathrm{N} 2,1.47\left(2 \mathrm{p}_{\mathrm{x}}\right)$ \\
\hline HOMO-24 & $2.83,5 \mathrm{~d}^{2}$ & $\mathrm{~N} 2,1.27\left(2 \mathrm{p}_{\mathrm{x}}\right), \mathrm{N} 2,1.04\left(2 \mathrm{p}_{\mathrm{y}}\right)$ \\
\hline HOMO-31 & $2.93,5 \mathrm{~d}_{\mathrm{xy}} ; 1.91,5 \mathrm{~d}_{\mathrm{yz}}$ & $\mathrm{N} 3,1.85\left(2 \mathrm{p}_{\mathrm{x}}\right) ; \mathrm{N} 1,1.22\left(2 \mathrm{p}_{\mathrm{y}}\right)$ \\
\hline HOMO-32 & $4.02,5 \mathrm{~d}_{\mathrm{xz}}$ & $\mathrm{N} 1,1.02\left(2 \mathrm{p}_{\mathrm{x}}\right)$ \\
\hline HOMO-35 & $6.98,5 \mathrm{~d}^{2}$ & $\mathrm{~N} 2,2.80\left(2 \mathrm{p}_{\mathrm{y}}\right) ; \mathrm{N} 1,1.21\left(2 \mathrm{p}_{\mathrm{x}}\right) ; \mathrm{N} 3,1.19\left(2 \mathrm{p}_{\mathrm{y}}\right)$ \\
\hline HOMO-38 & $2.32,5 \mathrm{~d}_{\mathrm{xy}}$ & $\mathrm{N} 2,5.64\left(2 \mathrm{p}_{\mathrm{x}}\right) ; \mathrm{N} 3,4.57\left(2 \mathrm{p}_{\mathrm{y}}\right) ; \mathrm{N} 3,4.57\left(2 \mathrm{p}_{\mathrm{z}}\right)$ \\
\hline HOMO-42 & $1.21,5 \mathrm{~d}_{\mathrm{z}}^{2}$ & $\mathrm{~N} 2,4.97\left(2 \mathrm{p}_{z}\right) ; \mathrm{N} 2,2.69\left(2 \mathrm{p}_{\mathrm{y}}\right) ; \mathrm{N} 2,1.98\left(2 \mathrm{p}_{\mathrm{x}}\right)$ \\
\hline
\end{tabular}




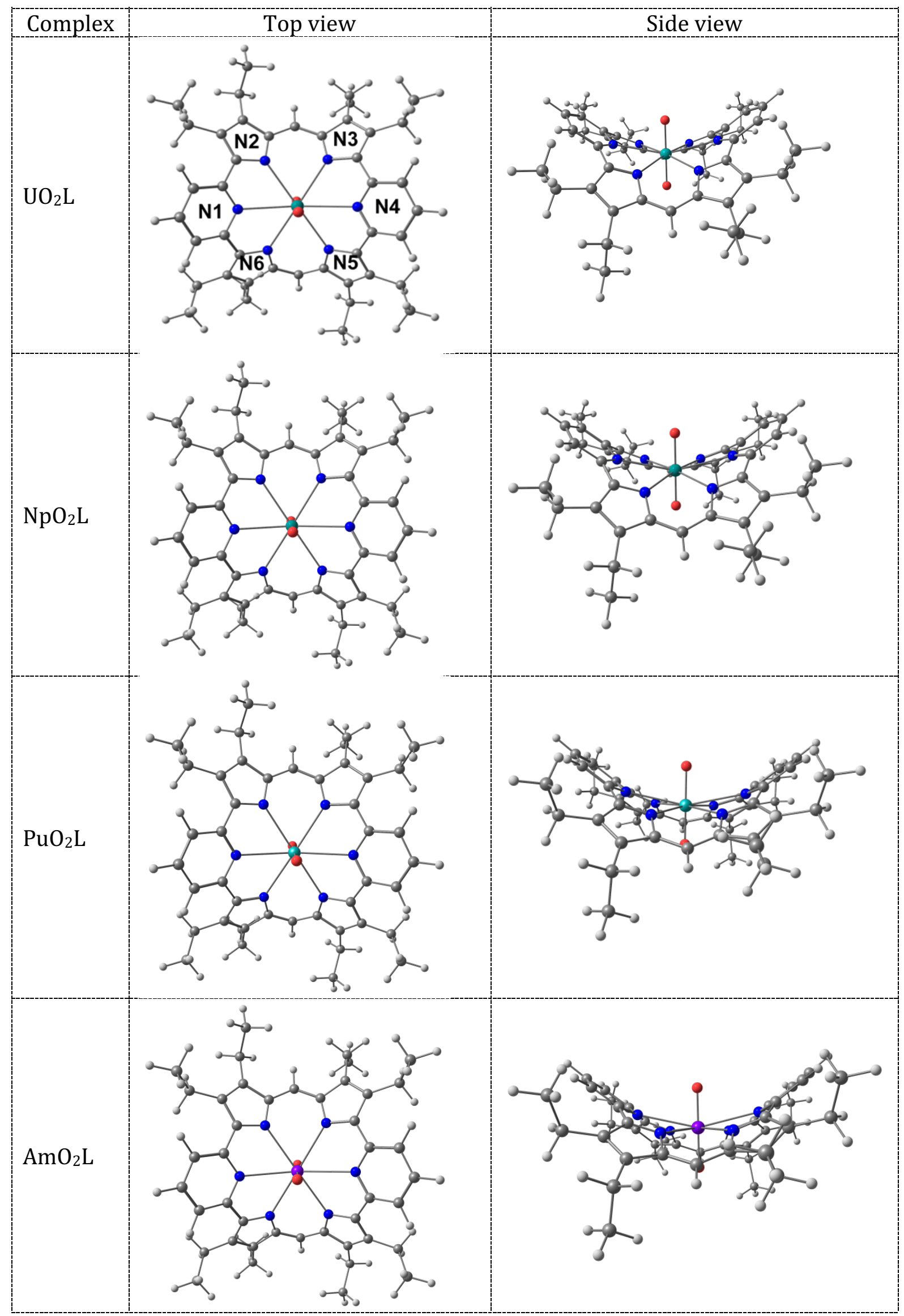




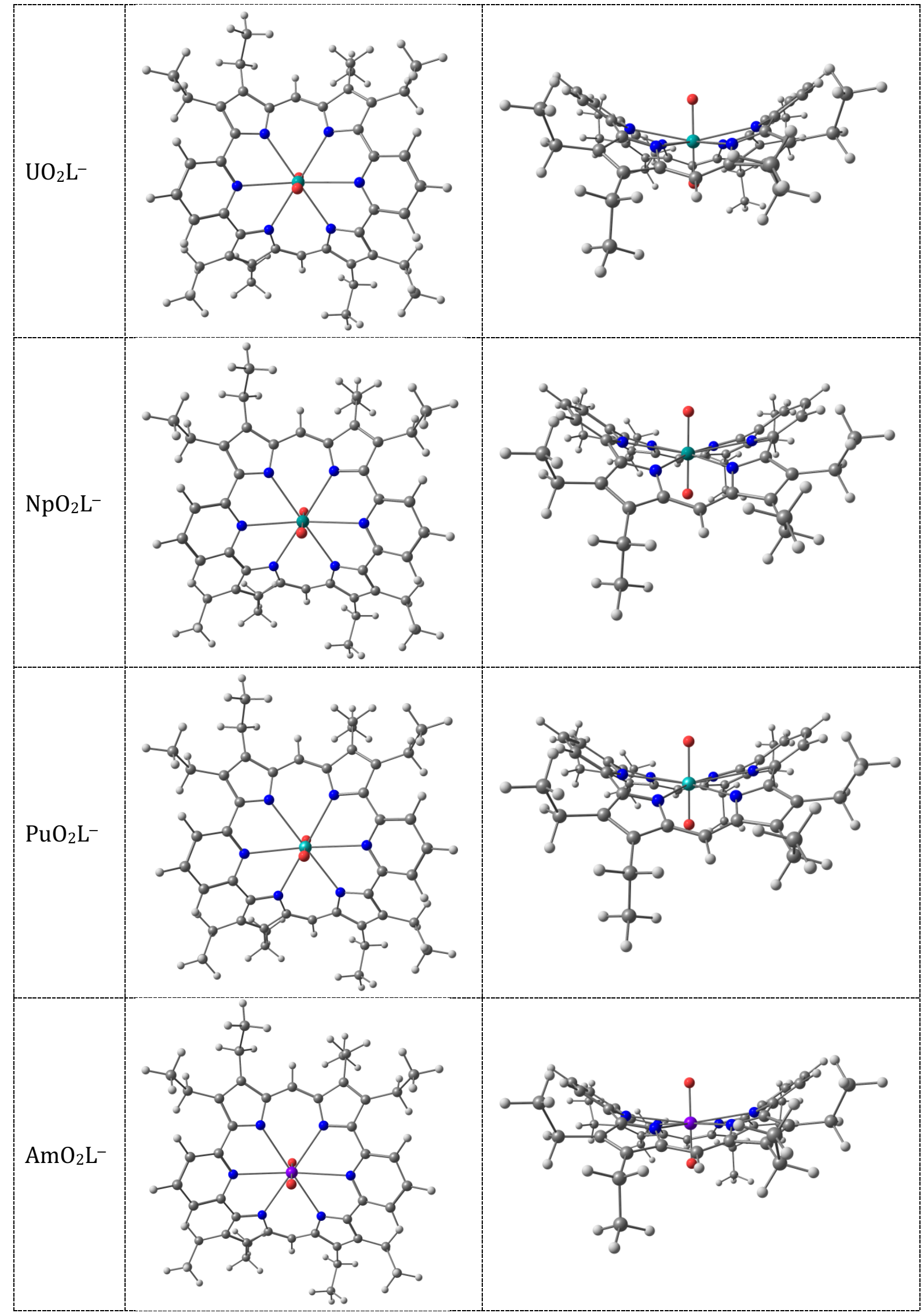

Figure S1: Optimized geometries of $\left[\mathrm{AnO}_{2}(\mathrm{~L})\right]^{0 / 1-}(\mathrm{An}=\mathrm{U}, \mathrm{Np}, \mathrm{Pu}, \mathrm{Am})$ complexes. 

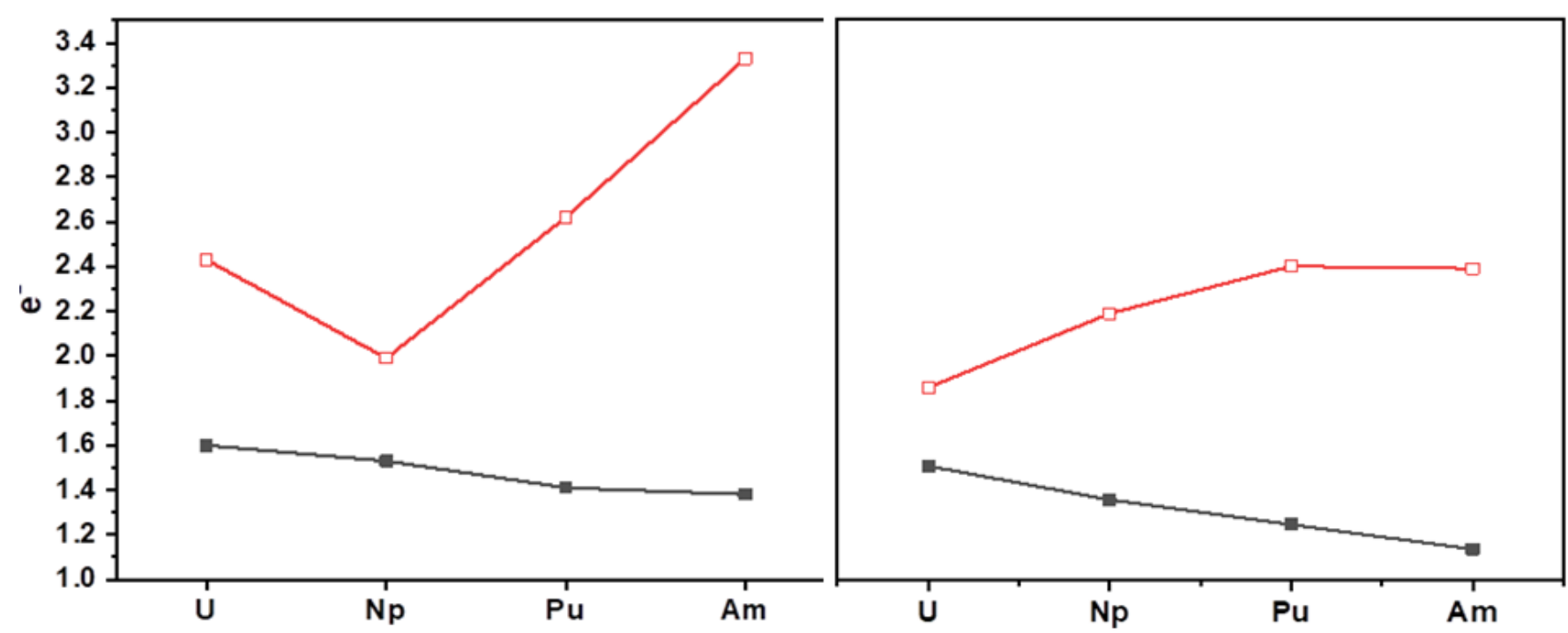

Figure S2: Electron donation into the valence d ( $\square$ ) and f ( $\square$ ) orbitals in An (VI) (left) and An (V) (right) complexes. 

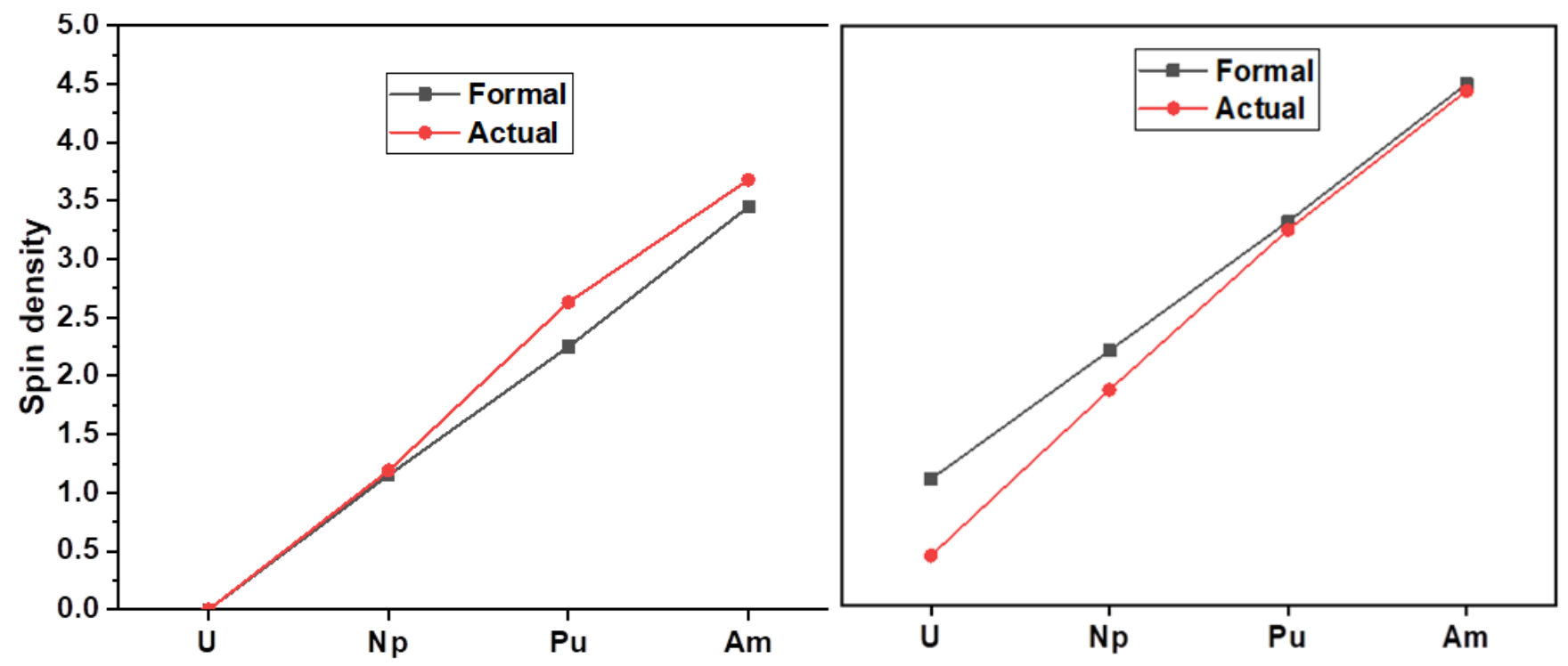

Figure S3: Formal and actual spin densities on the $\mathrm{An}(\mathrm{U}, \mathrm{Np}, \mathrm{Pu}$ and $\mathrm{Am}$ ) metal in the actinyl(VI) (left) and actinyl (V) (right) complexes. 


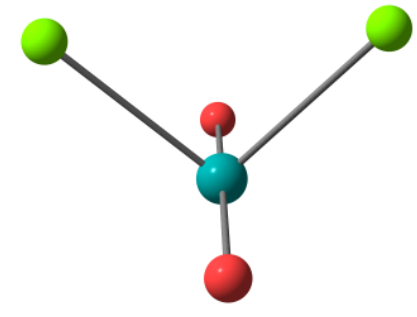

$\mathrm{UO}_{2} \mathrm{Cl}_{2}$

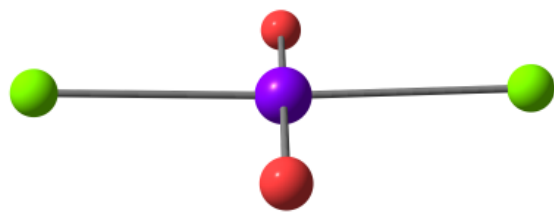

$\mathrm{AmO}_{2} \mathrm{Cl}_{2}$

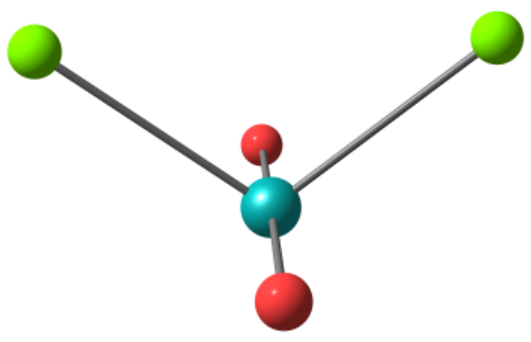

$\mathrm{PuO}_{2} \mathrm{Cl}_{2}^{-}$

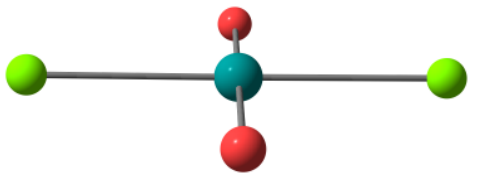

$\mathrm{NpO}_{2} \mathrm{Cl}_{2}$

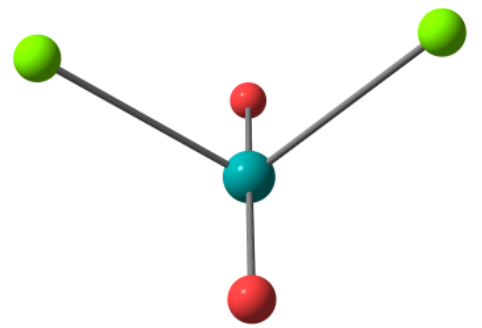

$\mathrm{UO}_{2} \mathrm{Cl}_{2}{ }^{-}$

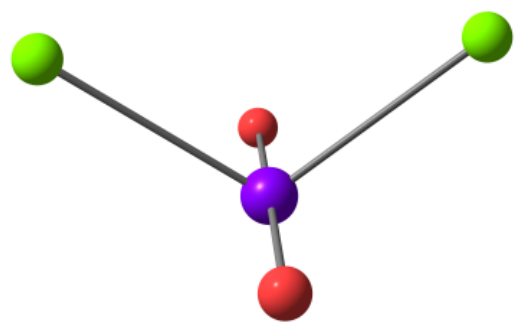

$\mathrm{AmO}_{2} \mathrm{Cl}_{2}^{-}$

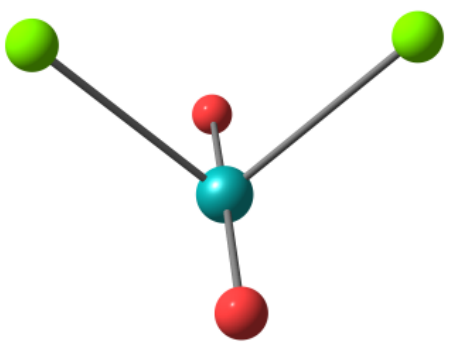

$\mathrm{PuO}_{2} \mathrm{Cl}_{2}$

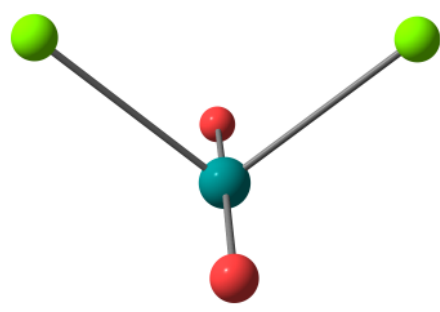

$\mathrm{NpO}_{2} \mathrm{Cl}_{2}-$

Figure S4: Optimized geometries of actinyl dichlorides at PBE/TZP level of theory in $\mathrm{CH}_{2} \mathrm{Cl}_{2}$. Red and green spheres represent $\mathrm{O}$ and $\mathrm{Cl}$ respectively. 


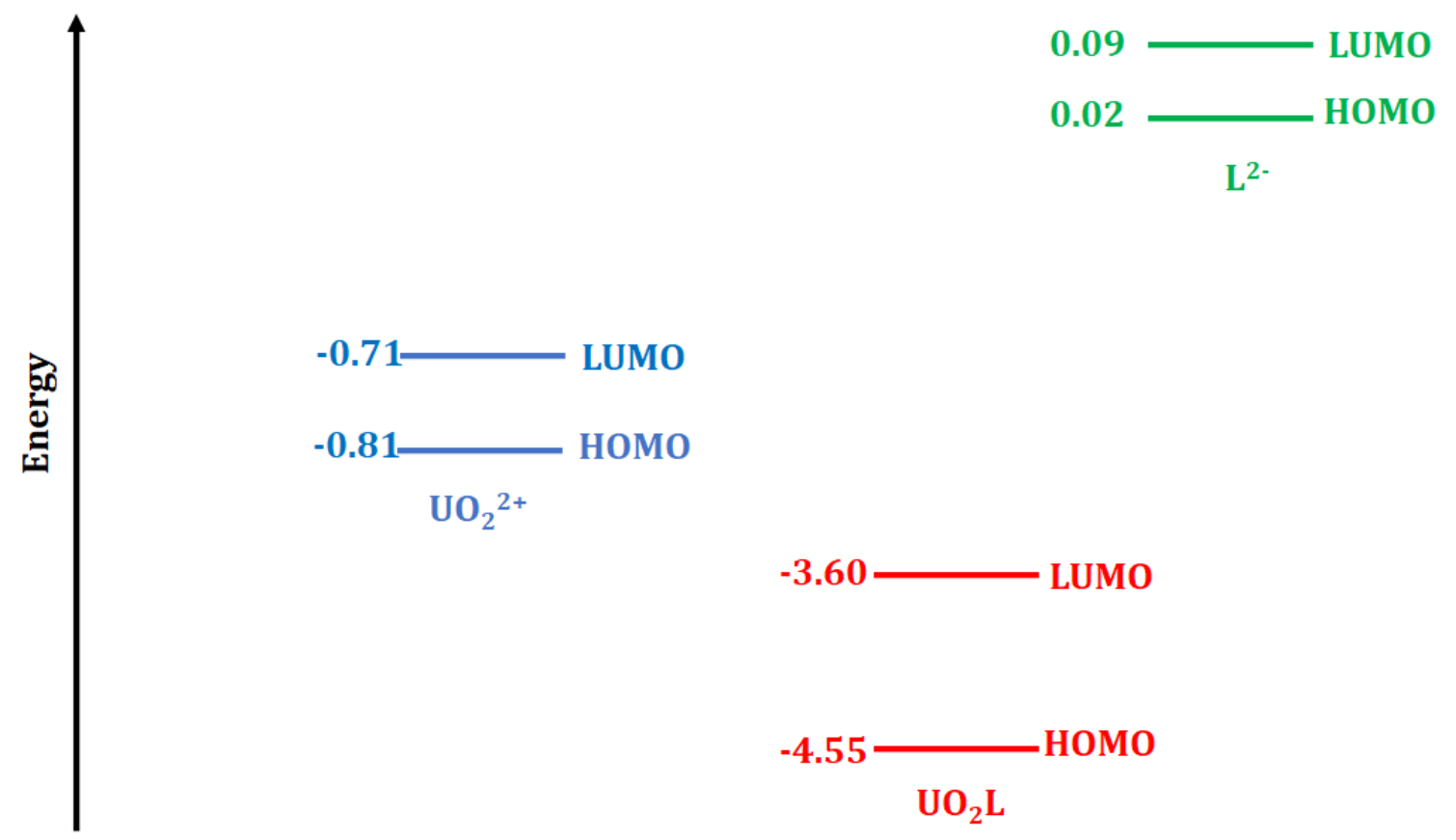

Figure S5: Molecular orbital energy $(\mathrm{eV})$ diagram of $\mathrm{UO}_{2} \mathrm{~L}$ and its two fragments. 